\title{
Modelling and determination of the transmission contact rate for contagious bovine pleuropneumonia
}

\author{
T. BALENGHIEN*, K. CHALVET-MONFRAY, D. J. BICOUT*AND P. SABATIER \\ Unité de Biomathématiques et Epidémiologie, INRA-Ecole Nationale Vétérinaire de Lyon, 1 avenue Bourgelat, \\ B.P. 83, 69280 Marcy l'Etoile, France
}

(Accepted 18 November 2004)

\section{SUMMARY}

Contagious bovine pleuropneumonia (CBPP) is a cattle respiratory disease that represents one of the major threats to cattle health and production in sub-Saharan Africa. The transmission contact rate of CBPP plays a key role in the spreading dynamics of the disease. We have developed an approach based on the combination of a SEIR model describing the spread of CBPP with the dynamic of seroconversion to determine the transmission contact rate for CBPP. This method has been subsequently applied to serological diagnostic data obtained from an experimental vaccine trial. As a result, we find that the transmission contact rates for subclinical, clinical and chronic infective states are respectively, $0 \cdot 084 / N, 0 \cdot 45$ and $0 \cdot 14 / N$ per animal per day, where $N$ is the herd population size, and the basic reproductive number corresponding to this trial $(N=28)$ is $R_{0}=27$.

\section{INTRODUCTION}

Contagious bovine pleuropneumonia (CBPP) is a respiratory disease caused by Mycoplasma mycoides subsp. mycoides Small Colony, designated $\mathrm{MmmSC}$ [1]. It is a disease classified in the A list of the World Organisation for Animal Health (ex. OIE) as causing cattle mortality and loss of production along with high costs for controlling [2]. Although CBPP outbreaks occur regularly in Europe, CBPP remains one of the major threats to cattle health and production in Africa. The cessation of pan-African campaigns of mass vaccination (PARC programme) has led to the re-emergence of the disease in sub-Saharan Africa [3].

CBPP is mainly transmitted through expectorations of coughing animals and secondarily through direct contact [4]. As well as being contagious for the

\footnotetext{
* Correspondence may be addressed to either author. (Email: t.balenghien@vet-lyon.fr)

(Email: dbicout@vet-lyon.fr)
}

duration of the incubation period, which is still debated, freshly infected animals also become contagious well before the onset of symptoms [5]. The clinical patterns are extremely varied, from asymptomatic to hyperacute forms. During the acute phase, which is the most contagious stage, animals undergo an increase of temperature, anorexia and respiratory difficulties, sometimes until death, and present lesions of severe fibrinous pneumonia with pleural exudate. Survivors of acute CBPP evolve to the chronic stage that is often asymptomatic. It is assumed that the chronic form is responsible for the disease persistence [6], but to an extent which is still unknown [7]. Moreover, some animals may have developed natural resistance to the disease.

In this paper, we address the issue of the transmission contact rate $(\beta)$, a parameter that plays a key role in the spreading dynamics of the disease since it measures the capability that an infective animal has of transmitting the disease to others. All outputs of models used in the control strategies of the disease 


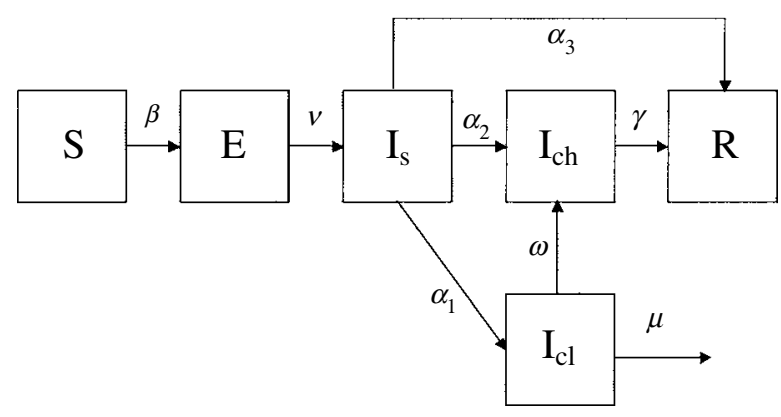

Fig. 1. Kinetic scheme of the within-herd compartmental model for CBPP.

and in cost-benefit studies are very dependent upon how the disease is spread, and thus, on the transmission contact rate. Unfortunately, estimated values of $\beta$ for CBPP are still lacking in the literature. For this purpose, we have developed an approach based on the combination of a SEIR model describing the spread of the disease with the dynamic of seroconversion to determine the transmission contact rate of CBPP. Indeed, rather than directly following the spread dynamics of a disease, which may prove to be an onerous (if not impossible) task even in experimental trials, serological diagnostics is becoming a widely used alternative technique for timing the disease propagation in a given sample of the population. In the form of a feedback loop, our approach uses such a kind of serological diagnostic data to assess the value of $\beta$ that in turn controls the propagation of the disease following the SEIR picture. We have subsequently applied this method to serological data obtained from an experimental vaccine trial to determine the transmission contact rate of CBPP in experimental conditions.

\section{METHODS}

\section{Description of mathematical models}

The within-herd spread of CBPP is described using the classical SEIR model where S, E, I and R represent the number of susceptible, latently infected, infective and recovered animals respectively [8]. In our model (Fig. 1), the infective class is, according to the epidemiology of CBPP, subdivided into three states: $I_{s}, I_{c l}$ and $I_{c h}$, where $I_{s}$ denotes the number of early infective animals at the end of the incubation period (subclinical), $\mathrm{I}_{\mathrm{cl}}$ the infective animals with clinical symptoms (cough) and $I_{c h}$ the infective animals in the chronic state [9]. Each of these infective states has a different infectiousness represented by a constant transmission contact rate, $\beta_{\mathrm{i}}$. The time evolution of CBPP infection within a herd is described by the following system of equations:

$$
\left.\begin{array}{l}
\frac{\mathrm{dS}}{\mathrm{d} t}=-\lambda \mathrm{S}=-\left(\beta_{\mathrm{I}_{\mathrm{cl}}} \mathrm{I}_{\mathrm{cl}}+\beta_{\mathrm{I}_{\mathrm{s}}} \mathrm{I}_{\mathrm{S}}+\beta_{\mathrm{I}_{\mathrm{ch}}} \mathrm{I}_{\mathrm{ch}}\right) \mathrm{S} \\
\frac{\mathrm{dE}}{\mathrm{d} t}=\lambda \mathrm{S}-\nu \mathrm{E} \\
\frac{\mathrm{d} \mathrm{I}_{\mathrm{s}}}{\mathrm{d} t}=\nu \mathrm{E}-\left(\alpha_{1}+\alpha_{2}+\alpha_{3}\right) \mathrm{I}_{\mathrm{s}} \\
\frac{\mathrm{d} \mathrm{I}_{\mathrm{cl}}}{\mathrm{d} t}=\alpha_{1} \mathrm{I}_{\mathrm{s}}-(\omega+\mu) \mathrm{I}_{\mathrm{cl}} \\
\frac{\mathrm{d} \mathrm{I}_{\mathrm{ch}}}{\mathrm{d} t}=\alpha_{2} \mathrm{I}_{\mathrm{s}}+\omega \mathrm{I}_{\mathrm{cl}}-\gamma \mathrm{I}_{\mathrm{ch}} \\
\frac{\mathrm{dR}}{\mathrm{d} t}=\alpha_{3} \mathrm{I}_{\mathrm{S}}+\gamma \mathrm{I}_{\mathrm{ch}}
\end{array}\right\}
$$

where $\lambda(t)$ is the force of infection, i.e. the fraction of susceptible population that the infective animals are able to enter in contact with and infect per unit of time; the other parameters are defined in the Table. In writing the system of equation (1), we have neglected the natural births and deaths and considered only the disease-induced mortality such that the total number of animals in the herd,

$N(t)=\mathrm{S}(t)+\mathrm{E}(t)+\mathrm{I}_{\mathrm{s}}(t)+\mathrm{I}_{\mathrm{cl}}(t)+\mathrm{I}_{\mathrm{ch}}(t)+R(t)$

decays as, $\mathrm{d} N / \mathrm{d} t=-\mu \mathrm{I}_{\mathrm{cl}}$.

In practice, except for clinical animals, a clear-cut distinction between SEIR states as described above is difficult, if not impossible. On the contrary, serological diagnostics is a widely used technique to identify animals that have been infected by pathogens. In this respect, it is very useful for practical purposes to complement the above equations with the dynamics of seroconversion. Animals newly contaminated by $\mathrm{MmmSC}$ become seropositive after a time, $T_{1}$, required for the synthesizing of antibodies. In the absence of additional infectious contacts, a seropositive animal will lose antibodies and become seronegative beyond an elapsed time, $T_{2}$, several months after the onset of antibodies. We consider, for simplicity, the case where the serological test is perfect (i.e. the sensitivity and specificity level is $100 \%$ ) and denote by $\mathrm{A}_{-}$the number of animals that are newly infected but still free of antibodies (i.e. those entering the E compartment of SEIR as described above) and denote by $\mathrm{A}_{+}$the number of animals that have seroconverted (i.e. carrying antibodies). The dynamics of seroconversion can be 
Table. Numerical values of the parameters of the model

\begin{tabular}{lllll}
\hline \hline Definitions & Symbol & Value & Unit & Reference \\
\hline Latent rate & $v$ & $0 \cdot 0714$ & $1 /$ day & {$[5]$} \\
Transmission contact rate of clinic & $\beta_{0}$ & $0 \cdot 45(0 \cdot 42-0 \cdot 46)^{\mathrm{a}}$ & $1 /$ animal/day & \\
Scaling factor of the contact rate & $\sigma_{0}$ & $0 \cdot 91(0 \cdot 83-1)^{\mathrm{a}}$ & & \\
Transition rate & $\alpha_{1}$ & $0 \cdot 0184$ & $1 /$ day & {$[4,10]$} \\
$\quad$ Subclinical to clinical & $\alpha_{2}$ & $0 \cdot 0422$ & $1 /$ day & {$[4,10]$} \\
$\quad$ Subclinical to chronic & $\alpha_{3}$ & $0 \cdot 0107$ & $1 /$ day & {$[4,10]$} \\
$\quad$ Subclinical to recovered & $\omega$ & $0 \cdot 0219$ & $1 /$ day & {$[10,11]$} \\
$\quad$ Clinical to chronic & $\mu$ & $0 \cdot 0257$ & $1 /$ day & {$[10,11]$} \\
Per capita mortality rate & $\gamma$ & $0 \cdot 0083^{\mathrm{b}}$ & $1 /$ day & \\
Recovery rate & & $\left\{13 \cdot 8^{\mathrm{c}}\right.$ & $\%$ & \\
Mortality and morbidity & $13 \cdot 7^{\mathrm{d}}$ & $25 \cdot 5^{\mathrm{c}}$ & $\%$ & \\
$\quad$ Deaths & $25 \cdot 7^{\mathrm{d}}$ & $71 \cdot 2^{\mathrm{c}}$ & $\%$ & \\
Cumulative clinical incidence & & $71 \cdot 3^{\mathrm{d}}$ & days & \\
Cumulative chronic incidence & & $20 \pm 4^{\mathrm{a}}$ & & \\
Time of appearance of antibodies & $T_{1}$ & &
\end{tabular}

a Result from the best fit to experimental data.

b See discussion in the text.

c Result from the experimental vaccine trial.

d Result from simulations.

described as:

$$
\left.\begin{array}{rl}
\frac{\mathrm{dA}_{-}}{\mathrm{d} t}= & \lambda(t) \mathrm{S}(t)-\lambda\left(t-T_{1}\right) \mathrm{S}\left(t-T_{1}\right) \\
\frac{\mathrm{dA}_{+}}{\mathrm{d} t}= & \lambda\left(t-T_{1}\right) \mathrm{S}\left(t-T_{1}\right) \\
& -\lambda\left(t-T_{1}-T_{2}\right) \mathrm{S}\left(t-T_{1}-T_{2}\right)
\end{array}\right\}
$$

with $\lambda(t)=0$ for $t \leqslant 0$. Note that these equations can also be used for IgM or IgG antibodies with corresponding $T_{1}$ and $T_{2}$. It is clear from equation (2) that the dynamics of seroconversion is related to the transmission contact rates through the force of infection, thus providing a useful way to determine the rate of $\beta$. These transmission contact rates play a key role in the spreading dynamics of the disease. Indeed, following the introduction of a single infectious animal into a herd entirely constituted of susceptible animals, CBPP will only spread if the basic reproductive number, $R_{0}$, is greater than 1 , i.e. $R_{0}>1$, where:

$$
\begin{aligned}
R_{0}= & \frac{\beta_{\mathrm{I}_{\mathrm{s}}} N_{0}}{\alpha}+\frac{\alpha_{1}}{\alpha} \frac{\beta_{\mathrm{I}_{\mathrm{cl}}} N_{0}}{(\mu+\omega)}+\frac{\alpha_{1}}{\alpha} \frac{\omega}{(\mu+\omega)} \frac{\beta_{\mathrm{I}_{\mathrm{ch}}} N_{0}}{\gamma} \\
& +\frac{\alpha_{2}}{\alpha} \frac{\beta_{\mathrm{I}_{\mathrm{ch}}} N_{\mathbf{0}}}{\gamma},
\end{aligned}
$$

with $\alpha=\alpha_{1}+\alpha_{2}+\alpha_{3}$. By definition, $R_{0}$ is the mean number of secondary cases generated by one primary case in a naive population. Thus, the disease will die out for $R_{0} \leqslant 1$. The expression of $R_{0}$ given in equation (3), that can also be derived using the next generation method [12], is the summation of three terms each representing the number of secondary cases generated by subclinical, clinical and chronic infective animals respectively. All these numbers of secondary cases are proportional to their respective $\beta$ 's.

\section{Model of the transmission contact rate}

The transmission contact rate, $\beta$, is the probability per unit of time that an infectious animal will have contact with and successfully transmit infection to a susceptible animal. It is a bi-individual rate (i.e. involves two individuals) that captures the aetiology of the infectious process and combines a number of epidemiological and environmental factors that affect the disease transmission. Under the homogeneous mixing hypothesis, the transmission contact rate, $\beta_{\mathrm{i}}$, of CBPP by an animal in one of the three infectious states can be factorized as $\beta_{\mathrm{i}}=a_{\mathrm{i}} \sigma_{\mathrm{i}} b_{\mathrm{i}}$, where $a_{\mathrm{i}}$ is related to the titre level of $\mathrm{MmmSC}$ in the animal's respiratory system, $\sigma_{\mathrm{i}}$ is the contact rate between an infective and a susceptible animal, and $b_{\mathrm{i}}$ the transmission probability in an established contact. As CBPP can be transmitted either indirectly through 
aerosol during animal coughing or through direct contact, the contact rate function of clinical animals (which cough) comprises two contributions, say, $\sigma_{\mathrm{I}_{\mathrm{cl}}}=\sigma_{a}+\sigma_{\mathrm{d}}$ (where $\sigma_{\mathrm{a}}$ and $\sigma_{\mathrm{d}}$ are the aerosol and direct contact rates respectively) while that of subclinical and chronic animals involve only $\sigma_{\mathrm{d}}$. At the scale of a herd, $\sigma_{\mathrm{d}}$ represents contributions to contagion of the very local contacts while $\sigma_{\mathrm{a}}$ stands for long-range contacts. It follows that $\beta_{\mathrm{I}_{\mathrm{s}}}=a_{\mathrm{I}_{\mathrm{s}}} \sigma_{\mathrm{d}} b_{\mathrm{I}_{\mathrm{s}}}$, $\beta_{\mathrm{I}_{\mathrm{cl}}}=a_{\mathrm{I}_{\mathrm{cl}}}\left(\sigma_{\mathrm{a}}+\sigma_{\mathrm{d}}\right) b_{\mathrm{I}_{\mathrm{cl}}}$ and $\beta_{\mathrm{I}_{\mathrm{ch}}}=a_{\mathrm{I}_{\mathrm{ch}}} \sigma_{\mathrm{d}} b_{\mathrm{I}_{\mathrm{ch}}}$. It makes sense to think that these $\beta$ 's are not independent of each other. In this respect, we set $\beta_{\mathrm{I}_{\mathrm{cl}}}=\beta_{0}$, and assuming that transmission probabilities of $\mathrm{MmmSC}$ are identical for all infectious states, i.e. $b_{\mathrm{I}_{\mathrm{s}}}=b_{\mathrm{I}_{\mathrm{cl}}}=b_{\mathrm{I}_{\mathrm{ch}}}$, then $\beta_{\mathrm{i}}$ can be written as follows: $\beta_{\mathrm{i}}=a_{\mathrm{i}} \sigma_{\mathrm{i}} b_{\mathrm{i}}$.

$\left.\begin{array}{l}\beta_{\mathrm{I}_{\mathrm{s}}}=\frac{a_{\mathrm{I}_{\mathrm{s}}}}{a_{\mathrm{I}_{\mathrm{cl}}}} \frac{\sigma_{\mathrm{d}}}{\sigma_{\mathrm{d}}+\sigma_{\mathrm{a}}} \beta_{0}=\frac{1}{5} \frac{\sigma_{0}}{N} \beta_{0} \\ \beta_{\mathrm{I}_{\mathrm{cl}}}=a_{\mathrm{I}_{\mathrm{cl}}}\left(\sigma_{a}+\sigma_{\mathrm{d}}\right) b_{\mathrm{I}_{\mathrm{cl}}}=\beta_{0} \\ \beta_{\mathrm{I}_{\mathrm{ch}}}=\frac{a_{\mathrm{I}_{\mathrm{ch}}}}{a_{\mathrm{I}_{\mathrm{cl}}}} \frac{\sigma_{\mathrm{d}}}{\sigma_{\mathrm{d}}+\sigma_{\mathrm{a}}} \beta_{0}=\frac{3}{8} \frac{\sigma_{0}}{N} \beta_{0}\end{array}\right\}$

The ratios $a_{\mathrm{I}_{\mathrm{s}}} / a_{\mathrm{I}_{\mathrm{cl}}}=1 / 5$ and $a_{\mathrm{I}_{\mathrm{ch}}} / a_{\mathrm{I}_{\mathrm{cl}}}=3 / 8$ have been estimated from the mean titre of $\mathrm{MmmSC}$ in the animal respiratory system during an experimental study of CBPP [5]. The ratios depend on the animal receptivity to a given $\mathrm{MmmSC}$ strain. Next, we assume that the transmission through the aerosol is densitydependent, i.e. $\sigma_{\mathrm{a}}$ is constant such that the force of infection due to aerosol contribution is proportional to $\sigma_{\mathrm{a}} \mathrm{I}_{\mathrm{cl}}$, and that the transmission through the direct contact is frequency-dependent, i.e. $\sigma_{\mathrm{d}} \propto 1 / N$ (where $N$ is the population size) in which the force of infection is a function of the proportion of infectivity, namely $\sigma_{\mathrm{d}} \mathrm{I} \propto \mathrm{I} / N$. The second equality in equation (4) is obtained by setting $\sigma_{\mathrm{d}} / \sigma_{\mathrm{a}}=\sigma_{0} / N$ and requiring that $\sigma_{0} / N<1$. With these definitions, we have $\sigma_{0}<N$, $\beta_{\mathrm{I}_{\mathrm{cl}}}$ is independent of $N$ but $\beta_{\mathrm{I}_{\mathrm{s}}}$ and $\beta_{\mathrm{I}_{\mathrm{ch}}}$ are both decreasing functions of $N$. Now, according to the relations in equation (4), the determination of three transmission contact rates is reduced to determine $\beta_{0}$ and $\sigma_{0}$, in for example, a vaccine trial.

\section{Experimental data}

For our purpose, we consider the experimental vaccine trial conducted in Australia by Hudson \& Turner [10] on 392 animals divided into 14 groups, each comprising 28 animals. In each group, the trial consisted of mixing nine non-vaccinated and 14 vaccinated animals with five experimentally infected and symptomless animals, and following up the daily seroconversion of non-vaccinated animals employing a complement fixation test [13]. As a result, the authors reported the daily proportion of seroconverted (IgM antibodies) animals, of animals with clinical symptoms, the proportion of deaths, and of animals with chronic lesions. The authors did not report either the spread dynamics of the disease or the entire serological dynamics, but only the day of the first positive serological reaction for each animal.

\section{Determination of the transmission contact rate}

In order to determine $\beta_{0}$ and $\sigma_{0}$ from the data described above, we ran simulations to mimic the situation of the vaccine trial. The program used for numerical simulations was written in Fortran language and compiled with Absoft Pro Fortran $8.0^{\mathrm{R}}$. Stochastic simulations are carried out following a birth-and-death process where each possible transition event (infection, seroconversion, recovery, death) occurs at random with rates given by the deterministic equation (1). All stochastic trajectories are run using the parameters given in the Table and with the initial conditions $\left(\mathrm{S}, \mathrm{E}, \mathrm{I}_{\mathrm{s}}, \mathrm{I}_{\mathrm{cl}}, \mathrm{I}_{\mathrm{ch}}, \mathrm{R}\right)=(9,0$, $5,0,0,14)$, where $\mathrm{S}$ represents the non-vaccinated animals, $I_{s}$ the infected ones, and the vaccinated animals are considered as recovered, i.e. $\mathrm{R}$.

A $\chi^{2}$ minimization procedure between the experimental data and an average of 1000 stochastic simulations has been used on the proportion of daily seroconversion to determine $T_{1}$ the onset time of antibodies, $\beta_{0}$ and $\sigma_{0}$. As a control, the simulated deaths and cumulative incidence for clinical and chronic animals are compared with observations of the experimental trial in the Table. Next, 14 trajectories (equivalent to 14 groups of the experimental trial) using the parameters just determined have been run to generate the curves in Figure 2.

\section{RESULTS AND DISCUSSION}

The best fit to experimental data using the $\chi^{2}$ minimization procedure gives $T_{1}=20$ days for the onset of antibodies, $\sigma_{0}=0.91(0.83-1)$ and $\beta_{0}=0.45(0.42-0.46)$, corresponding to $R_{0}=26 \cdot 5(25 \cdot 2-26 \cdot 7)$ calculated from equation (3) with $N=\mathrm{S}_{0}=9$. The transmission contact rates for infective animals are: $\beta_{\mathrm{I}_{\mathrm{s}}}=0.084 / N$, $\beta_{\mathrm{I}_{\mathrm{cl}}}=0.45$ and $\beta_{\mathrm{I}_{\mathrm{ch}}}=0 \cdot 014 / N$ per animal per day, where $N$ is the time-dependent herd population size. At the beginning of the experiment when 

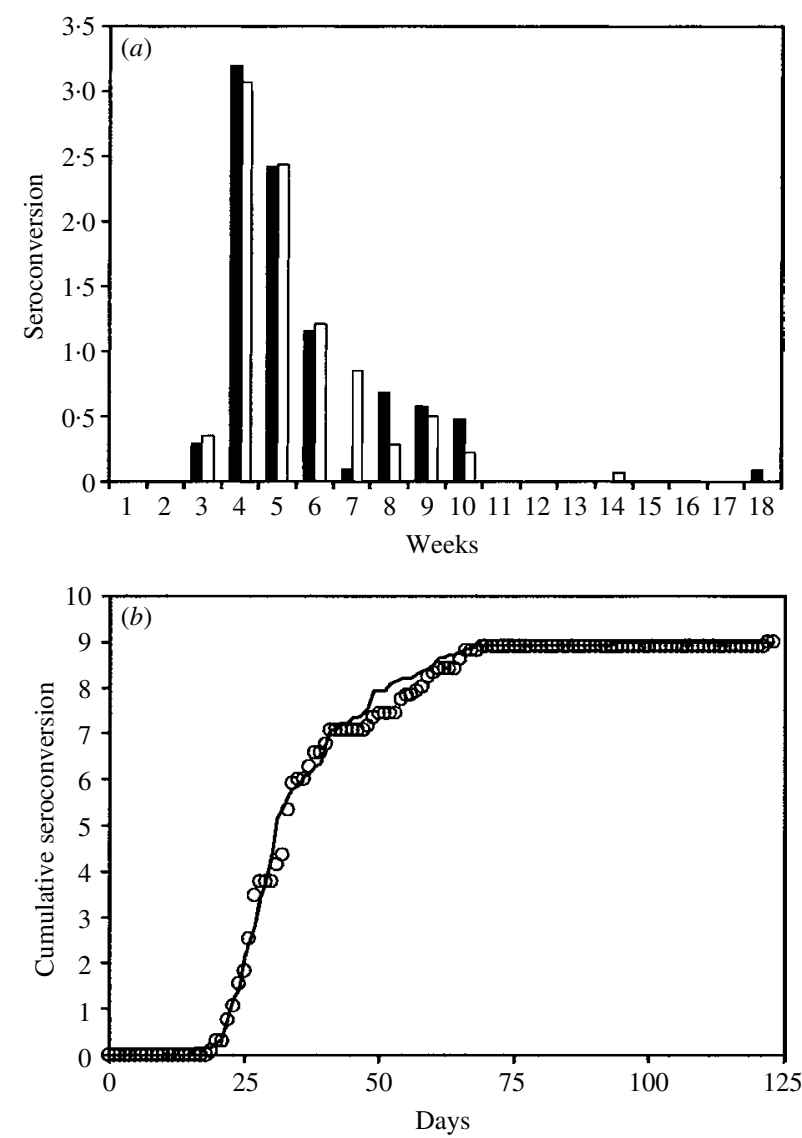

Fig. 2. Experimental data [(a) black histograms, $(b)$ symbols] from Hudson \& Turner [10] and simulated data [ $(a)$ white histograms, $(b)$ solid line] for a vaccine trial. Panel $(a)$ shows the incidence of weekly seroconversion and panel $(b)$ the cumulative daily seroconversion. The parameters used are given in the Table, with $\beta_{0}=0.45$ per animal per day, $\sigma_{0}=0.91$ and $T_{1}=20$ days.

$N=28$, we had ratios between $\beta_{\mathrm{I}_{\mathrm{cl}}} / \beta_{\mathrm{I}_{\mathrm{s}}}=150$ and $\beta_{\mathrm{I}_{\mathrm{cl}}} / \beta_{\mathrm{I}_{\mathrm{ch}}}=90$.

Simulated and experimental mortality and morbidity are respectively, $13.7 \%$ and $13.8 \%$ for deaths, $25.7 \%$ and $25.5 \%$ for cumulative clinical incidence and $71.3 \%$ and $71.2 \%$ for cumulative chronic incidence (see Table).

Under the hypotheses that (i) all I classes are infective with a given $\beta_{\mathrm{i}}$ and (ii) $\beta_{\mathrm{i}}$ 's are related to each other as given in equation (4), we found that the transmission contact rates of CBPP are approximately $10^{-3}-10^{-1}$ per animal per day. However, it is worthwhile to underline that these determined values have been obtained for experimental conditions that are known to be challenging for infectious contacts with a high degree of confinement and highly virulent strain of $M m m S C$. For instance, the mean number, $R_{0}$, of secondary cases generated by an infective animal in this experimental condition is $R_{0}=27$, which is of the same order as the population size $N=28$ of the trial. Thus, one expects that the transmission rates found in field and natural conditions be approximately one- to twofold smaller than in experimental conditions. As an illustrative comparison, the transmission contact rate for tuberculosis (another chronic respiratory disease) has been found to be $\sim 10^{-5}$ per animal per day for a herd of a few hundred animals in natural conditions $[14,15]$.

Values of transmission contact rates obtained above are conditional to the duration of the chronic state. The reason why chronic animals are considered to be responsible for the persistence of the disease is that active MmmSC can be found in the sequestra of chronic animals during 27 months [7]. In our simulations, we found that the spreading dynamics became independent of the chronic state for durations of $>30$ days (results not reported). Thus, consistent with the literature $[4,6]$, we used a mean duration of 120 days for the chronic state in all simulations. We also found that not just the duration but the presence of the chronic class is essential to correctly fit experimental data as the introduction of subclinical animals generates chronic ones according to the dynamic picture described earlier. When the chronic state is removed from the spreading dynamics of the disease, simulations failed to properly reproduce the time evolution of seroconversion and the values of transmission contact rates thus obtained are much higher.

Nevertheless, it is worthwhile mentioning that the same set of experimental data can be well fitted even with spreading dynamics models that do not incorporate the chronic class. Examples of such models use a distribution of incubation periods for the $\mathrm{E}$ class (Lesnoff, M. personal communication). Hence, different patterns of the spreading dynamics of the disease may also lead to identical dynamics of seroconversion. This emphasizes that more data on the dynamics of the disease are required to test and choose appropriate models.

Finally, it is already remarkable that the approach outlined above is capable of reproducing a number of features of an experimental trial. It will now be interesting to use the same method on serological field data to determine the transmission contact rates of CBPP in natural conditions, provided that the recovery rate is known. However, the model can be embellished in various directions, for instance, by simultaneously using a different functional form of $\beta$ vs. $N$ and 
including the distribution of incubation times. Such a work is underway.

\section{ACKNOWLEDGEMENTS}

The authors thank Matthieu Lesnoff (CIRADEMVT) for fruitful discussions. Financial support from DGA and CNES in the form of grants is gratefully acknowledged.

\section{REFERENCES}

1. Cottew GS, Yeats FR. Subdivision of Mycoplasma mycoides subsp. mycoides from cattle and goats into two types. Aust Vet J 1978; 54: 293-296.

2. Egwu GO, Nicholas RA, Ameh JA, Bashiruddin JB. Contagious bovine pleuropneumonia, an update. Vet Bull 1996; 66: 875-888.

3. Yaya A, Golsia R, Hamadou B, Amaro A, Thiaucourt F. Essai comparatif d'efficacité des deux souches vaccinales $\mathrm{T} 1 / 44$ et $\mathrm{T} 1 \mathrm{sr}$ contre la péripneumonie contagieuse bovine. Rev Elev Med Vet Pays Trop 1999; 52: 171-179.

4. Provost A, Perreau P, Breard A, Le Goff C, Martel JL, Cottew GS. Contagious bovine pleuropneumonia. Rev Sci Tech $1987 ; 6$ : 625-679.

5. Belli P, Poumarat F, Perrin M, Longchambon D, Martel JL. Reproduction expérimentale et évolution de la péripneumonie contagieuse bovine dans un groupe de bovins et de caprins: aspects anatomocliniques. Rev Elev Med Vet Pays Trop 1989; 42: 349-356.

6. Masiga WN, Domenech J, Windsor RS. Manifestation and epidemiology of contagious bovine pleuro- pneumonia in Africa. Rev Sci Tech 1996; 15: 1283 1308.

7. Windsor RS, Masiga WN. Investigations into the role of carrier animals in the spread of contagious bovine pleuropneumonia. Res Vet Sci 1977; 23: 224-229.

8. Anderson RM, May RM. The basic model: dynamics. In: Infectious diseases of humans. Dynamics and control. Oxford Science Publications; 1985: 122-143.

9. Lesnoff M, Laval G, Bonnet P, Chalvet-Monfray K, Lancelot R, Thiaucourt F. A mathematical model of the effects of chronic carriers on the within-herd spread of contagious bovine pleuropneumonia in an African mixed crop-livestock system. Prev Vet Med 2004; 62: 101-117.

10. Hudson JR, Turner AW. Contagious bovine pleuropneumonia: a comparison of the efficacy of two types of vaccine. Aust Vet J 1963; 39: 373-385.

11. Martel JL, Belli P, Perrin M, Dannacher G, Poumarat F. La péripneumonie bovine contagieuse. Rec Méd Vét 1985; 161 : 1105-1113.

12. Diekmann O, Heesterbeek JAP. Mathematical epidemiology of infectious diseases. John Wiley \& Son, 2000.

13. Campbell AD, Turner AW. Studies on contagious pleuropneumonia of cattle. IV. An improved complement-fixation test. Aust Vet J 1953; 29: 154-163.

14. Barlow ND, Kean JM, Hickling G, Livingstone PG, Robson AB. A simulation model for the spread of bovine tuberculosis within New Zealand cattle herds. Prev Vet Med 1997; 32: 57-75.

15. Perez AM, Ward MP, Ritacco V. Simulation-model evaluation of bovine tuberculosis-eradication strategies in Argentine dairy herds. Prev Vet Med 2002; 54: 351-360. 